\title{
Visual Discourse of Comics in English Language Teaching
}

\author{
Razvan Radan \\ Sarasas Ektra School, Thailand \\ e-mail: razvanradan@hotmail.com
}

\begin{abstract}
This article discusses the findings of theoretical research on visual communication and comic book discourse that aimed to identify viable approaches for English language teaching based on comics. Comics are one type of creative text that can have the same complexities or intricacies as for example a novel. One problem identified in SLT is that while creative texts are used in teaching, the focus is on the written word - novels, short stories, poems. Few if any teaching approaches promote the use of comics in the classroom environment. If comics are suggested as teaching materials, the recommended approach is very similar to that used when dealing with non-visual texts. As a consequence, students tend to ignore the images and thus miss out a possible avenue that can help with meaning making. There is then a need for a better understanding of how visual communication works in comics and how meaning is created from the interplay of image and text. This article examines how visual communication happens, what are the elements of a visual narrative and then continues to identify the elements that form the comic book discourse. Using a framework based on the theories that identify and explain the workings of the structural elements of a panel as well as the social function of visual communication this researcher discusses how comics communicate to their readers. The findings suggest that in order to develop a methodology and to successfully use comics in teaching, the educator needs to rethink the current practices when dealing with comics or any other "image + word" texts. Image and text cannot be separated and must be treated as a whole; only then will comics reveal their full potential as
\end{abstract}


viable, creative teaching materials.

Keywords: visual learning, multimodality, ELT, SLA

\section{INTRODUCTION}

Textbooks have been created for the purpose of language teaching, graded readers were devised to help the learning process and even the use of authentic texts has been painted as the panacea to language acquisition, however, from the observed reality of the classroom, none seem to work well enough. Creative texts are employed in the teaching environment but rarely is there a clear reasoning behind their selection. More often than not, the focus has been on the written text - novels, short stories, poems. Comics belong to this group of creative texts however few, if any, teaching methodologies or curricula use or promote the use of comics in the classroom environment. Carey (2004) states in his book Going Graphic: Comics at Work in the Interactive Classroom that while there is plenty of professional literature available on SLA and on comics studies respectively, there are few studies that "look specifically at the intersection of the two areas: using comics as a vehicle for second language acquisition". (p. 25) What literature is available so far focuses mostly on what to do with comics in a classroom environment and the suggested approach is similar to that employed when dealing with traditional non-visual texts. One of the results of this approach is that learners overlook the visuals. For example Skorge's (2008) empirical study shows that more often than not learners employ "cognitive economy" (p. 274) which indicates the learner's unwillingness to go beyond a first reading of the image. Her finding is that learners do not expect visuals to be other than decorative and therefore they settle "on the first reading of the depicted objects and actions that springs to mind, without being concerned about the lack of overall coherence in the visual narrative that emerges". (p. 274) Another result of this study shows that once learners settle on an interpretation, it is impossible to change their perception of what is being shown in the visual. Skorge concludes that students need to be instructed in the use and interpretation of the visuals. This, on the grander scale, is beneficial to teach learners visual literacy as required by the modern world - the "mediasphere" (O'Brien, 2001 in Brozo, W \& Fynt, E.S, 2010, p. 526). Felten (2008) calls for the need to provide this type of education because "living in an image rich world ... does not mean students naturally possess sophisticated visual literacy skills, just as continually listening to an iPod does not teach a person to critically analyze or create music" (p. 60 cited in Brozo, W \& Fynt, E.S, 2010 p. 526). Goldstein (2016) also identifies a shift towards the visual in our current global culture and points out that while in the past language teaching materials treated the visual components as "merely decorative, without educational value" today more and more educators have to "evaluate not only the presentation of grammar and vocabulary or the amount and type of skills work, but the visual aspects as well." (p. 2) 
It is this researcher's strong belief that comics can also help to mitigate a lot of the problems present in ELT and offer a viable alternative to the traditional materials used in the classroom, especially in the Asian context. One of the problems inherent with learning a second language is learner motivation; while motivation per se does not make the principal object of this study, it should not be completely ignored. In a strong visual culture like Thailand's, comics can motivate a reluctant learner to read in the target language. Reading is just one of the skills being developed in a language class and a skill not isolated from the others. What is gained from reading can have a positive influence on, for example, writing and vocabulary. If a learner ignores one set of skills, the others will be affected. Because of the visual nature of the Thai society in particular, comic books have the ability to motivate students to read as they present a "sanook" (fun) outlook to an activity they are required to partake in order to acquire knowledge of a language. Provided the teacher makes the right selection, students can connect more easily with comics because of their artwork, pop culture elements, language use and novelty factor. A visual narrative may also help students create meanings and interpret texts. Meaning is not the domain of words alone, but also of line, shape, color, movement or pose. In other words, the artwork of a comic book is part of the language and meaning is created when the two are accessed together.

This article examines the way comics work and how meaning is created from the interplay of image and text. It looks at how comics as creative texts can work in an ELT classroom through the prism of visual communication and applied linguistics and aims to identify methodological approaches to English language teaching that can be developed based on the visual discourse of the comics medium.

\section{LITERATURE REVIEW}

This brief literature review will identify the elements that cartoonists use in creating the images that form the visual narrative sequences beginning with McClouds' pictorial vocabulary continuing to Cohn's structural elements of a comic and finally ending by looking at an image (a comic panel for example) from a functional perspective making use of Kress - van Leeuwen model based on Halliday's systemic functional grammar.

McCloud chooses the term "icon" to represent "person, place thing or idea". Icon includes symbols that represent "concepts, ideas and philosophies", language and finally pictures (that have various degrees of "realism"). Pictures are arranged from real to abstract, with abstract being divided into 2 further planes: the art object, the realm of color, shape, line and icon where every line or dot means something. Most pictures in comics belong to the iconic category because this way comic artists create visuals that lend them universality and that allows for self-identification by the reader. The opposite also holds true: realism makes pictures removed from the reader by having clear definition and so the otherness is emphasized.

While McCloud gives us a general taxonomy of visual elements based on their closeness (or lack of) to our perceived reality, Cohn (2013a) proposes a more indepth analysis and classification of the structural elements of comics. 
When drawing comics, open class items are made up of elements such as hands, fingers, eyes, and even a whole body. These elements are the actual carriers of meaning; they are culture bound and are either ingrained in the collective consciousness and as such repeated or are used in a novel way to establish new schemas to be used. These are the productive elements that make up the visuals.

The closed-class lexical items are the following: speech bubbles and balloons, indexical lines, impact stars and so on. These elements work like closed class lexical items in comics because, just like their counterparts in linguistics, they are used to serve a function (i.e. speech bubbles show that someone is speaking or impact stars show that something was hit); also, just like their counterparts in linguistics, the closed class lexical items identified by Cohn didn't change much throughout the times (speech bubbles can be traced back several hundreds of years in both Western and Asian visual narratives).

Apart from looking at the elements used to create the actual visuals in the panels of a comic, Cohn's research also gives us insight into how the human mind makes sense out of visual narratives by organizing sequential images into hierarchic constituents, analogous to the organization of grammatical categories in the syntax of sentences. When he tested out the brain's reaction to structural disruptions in the visual narrative sequence he observed similar responses to studies done on brain response to structural violations in language and music. (Cohn, 2014) The main elements of Cohn's visual narrative grammar are:

- orienter - panels that help create a context

- establisher - similar to orienters but the setting for the actual action is shown and/or the characters are introduced

- initial - are panels where the action begins

- prolongation - are panels that serve to create a sense of tension by amplifying a certain component of an action

- peak - these are the panels where the action is completed

- release - panels that show a passive state, the aftermath of an action or the coda.

All these elements put together create a "phase" which can be considered analogous to a phrase in literary language and which looks like this:

"Phase -> (Establisher) - (Initial (Prolongation)) - Peak - (Release)" (Cohn, 2013a) If phrases make up sentences then these phases make up an "arc" - "a visual sentence". Just as in verbal language, there are different ways to saying the same thing using the components of the visual language. A very short, simple visual narrative sequence can be created using an initial and a peak panel. We can expand on this by adding more panels to one or more parts of the arc. 


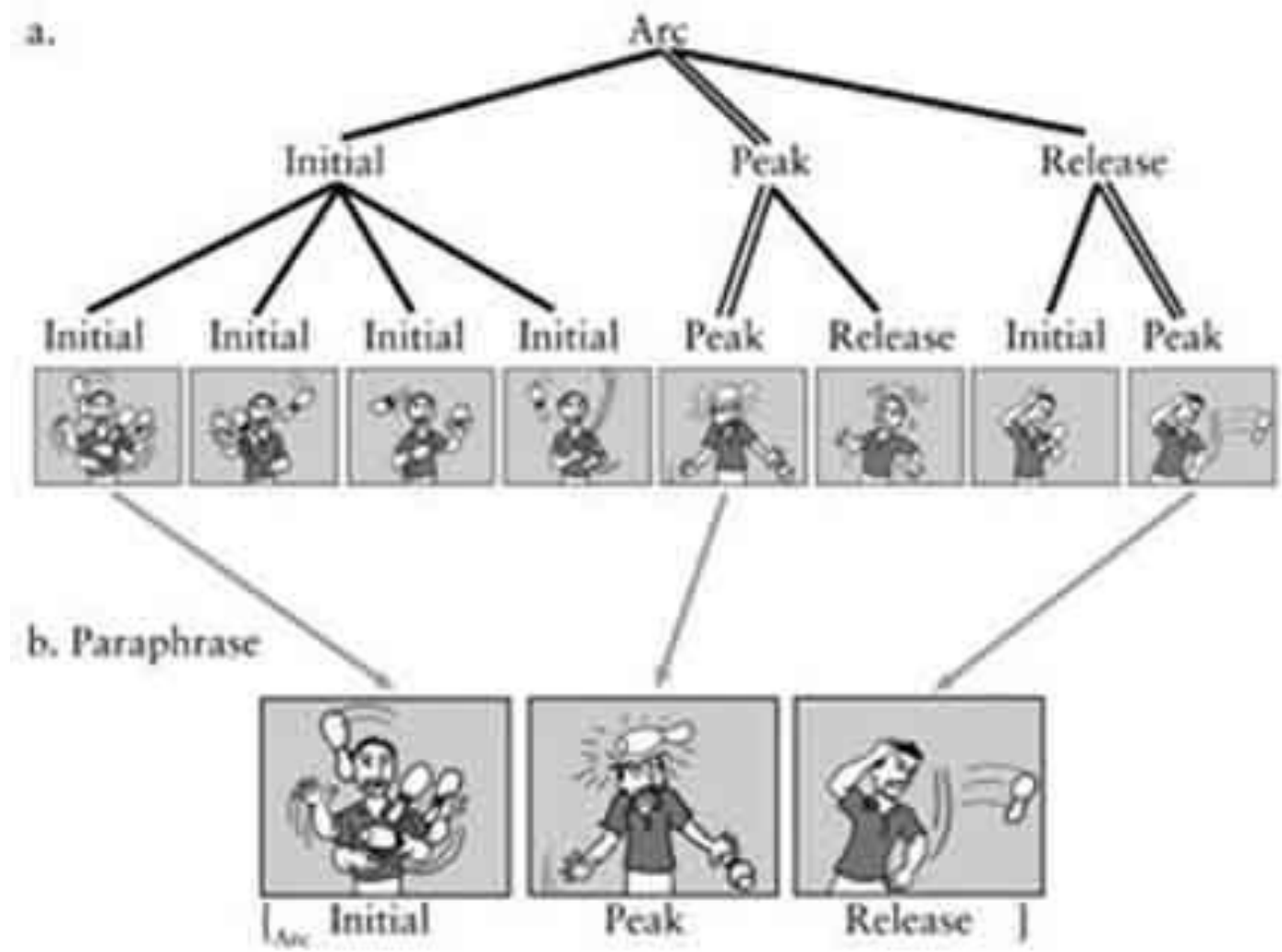

Fig. 1 - Embedding of phases and paraphrasing (Cohn, 2013a)

An example of how to create a complex visual narrative structure is shown in Figure 1.a. The initial is made up of 4 panels that all represent the same information, followed by two panels that together act as a peak followed by a conclusion in the last two panels which act as a release. This example shows that the narrative categories discussed previously can be used for discussing single panels or sequences of panels. Finally, Figure 1.b shows how to paraphrase a sequence of panels.

Moving on from a structural to a functional analysis of the visual narrative, a second way to look at how we make sense of comics is revealed. This approach implies using a model developed by Kress and van Leeuwen to read images, which in turn is based on Halliday's metafunctions of language.

The representational metafunction of an image defines the presentation of the elements that make up the image (participants - objects or elements, actions, setting). There are two possibilities of doing this in an image: using narrative representations or conceptual representations.

Narrative representations are images in which the composition creates a sense of action, images in which "participants are connected by a vector, they are represented as doing something to or for each other." (Kress, van Leeuwen, 2006; p.59) Narrative representations show a transient moment in time, an event that is unfolding or an action that is happening with the key element being the 'vector'. This 'vector' can be represented by a multitude of elements: diagonal lines, shapes 
that point to something; they can be concrete elements (a hand pointing, a tool in the hands of a participant, the gaze of a participant) or abstract like for example arrowheads in flowcharts.

Conceptual representations on the other hand are classificatory, analytical and symbolical. (p.59) These types of images are identified by the lack of an action, the objects or elements are stable; the participants are represented in terms of class, structure or meaning. Classificatory representations show participants as belonging to a taxonomy, to the same group; for example a panel in a comic depicting an assortment of weapons on a table, or a panel depicting an assorted group of rotten corpses. Analytical representations draw the viewer's attention towards the properties or attributes of a participant. In comics, an example of such a representation may be a panel showing a close-up of Superman standing tall atop a building: the viewer can examine his costume, his body shape, etc. Finally, symbolical conceptual representations are processes "about what a participant means or is". (p.105) Usually this is done in relation to an element that is otherwise appearing out of place in the image and then we are talking about symbolic attributive processes, like for example an apple in a Renaissance painting depicting the workshop of an inventor hinting at forbidden knowledge. Another way of creating a Symbolical conceptual representation is to use a suggestion and then we have what Kress and van Leeuwen call symbolic suggestive processes where meaning is shown as coming from within by use of for example color, hue, light.

The interactive metafunction of images is akin to the one relating to verbal languages as identified by Halliday. Broadly speaking, images can offer information to the viewer or demand a kind of response from the viewer (and usually with comics we are talking about an emotional response). If we look at the previous metafunctions, specifically the narrative and conceptual representations, we can infer that images where we have a narrative or where there is an absence of a gaze directed at the viewer can be considered as offer images and demand images are usually conceptual images that also incorporate a gaze directed at the viewer. Demand images ask the viewer to examine the particulars. Other things to consider when discussing the interactive metafunction of images is the framing of the images, the use of angles and perspective which all affect the interaction between the viewer and the image in terms of social distance, involvement and power (Kress, van Leeuwen, 2006; p.124-149; Eisner, 1985; p.88-97)

Finally, the compositional metafunction in Kress and van Leeuwen's model is the equivalent to the textual metafunction in Halliday's approach. It is the way in which the previous two functions, the representational and interactional, are combined to create the visual text. There are three elements that together determine these interactions that create the whole: information value (given or new, ideal or real), salience (size, color, placement in the frame) and framing. (p.177-204)

\section{RESEARCH METHODOLOGY}

In order to illustrate the applicability of the discussed theoretical framework, the article discusses sample panels from the following comics: Apollo 12 by Herge (creator of Tintin), Persepolis by Marjane Satrapi and Astroboy by Osamu Tezuka. 
Apollo 12 was chosen because the text and the language offer very similar meanings and this can help less proficient learners deal with the overall text. It can therefore be considered an "easy" text. The content of this 4 page comic is another reason for its selection: it tells the story of the Apollo 12 mission in a rather factual and informative way but without being pedantic. It then can be used to actually teach young learners about the early days of space exploration and therefore could be incorporated not just in an English class but also in a Science lesson.

The second comic selected for this analysis is the graphic novel Persepolis by Marjane Satrapi. It is the story of the author's childhood growing up in Iran at the time of the Islamic Revolution, her high school years in Vienna, her return home and the events that led to the author's decision to live in exile abroad. It is a graphic novel that on the surface seems simple and approachable. The content of this graphic novel however, is far from being simple: it deals with complex issues including freedom, political upheaval and religion to name just a few. It is then obvious that such a graphic novel would necessitate a more mature audience than the previous one afforded by the Apollo 12 comic. Because of its length, the discussion of Persepolis will be limited to selected panels only.

Finally, the inclusion of panels from the Japanese manga Astroboy will serve to illustrate that while it comes from a different geographical (and cultural) zone it nevertheless conforms to the same framework as the other two comics identified previously and the same lessons can be done with comics from all over the world.

Looking at these two types of discourse - textual and visual - one can make inferences of possible ways in which comics can be used effectively in ELT. To do this, a methodology is needed that can be generally applicable. In order to establish such an approach, this researcher will try to identify the following aspects: what is the role of the language, how does the learner fit in this theory and what is the role of the teacher.

\section{FINDINGS}

\subsection{A Functional Analysis of Panels Using Kress - van Leeuwen Model}

Herge's Apollo 12 was originally published in black and white in the French weekly Paris-Match No. 1073 on November 29 1969. The version used for this analysis is a color English translation. A quick reading of this text shows that most of the panels used to create the visual discourse of this comic show images that belong to the narrative representational category as identified in the Kress-van Leeuwen metafunction model of the visual language system. These types of panels are defined by a sense of action; the viewer can see a narrative taking place. In the example shown in Figure 2 the vector that creates the visual sense of action is the rocket itself which points away from planet Earth. Other techniques that are employed in the creation of this comic that create the narrative are the use of the diagonal lines, either to represent an object or to illustrate an entire panel. 


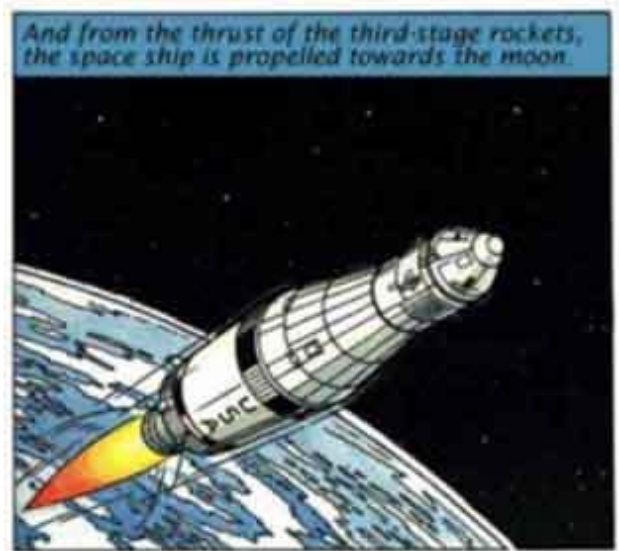

Fig. 2 - Apollo 12 (Herge, 1969)

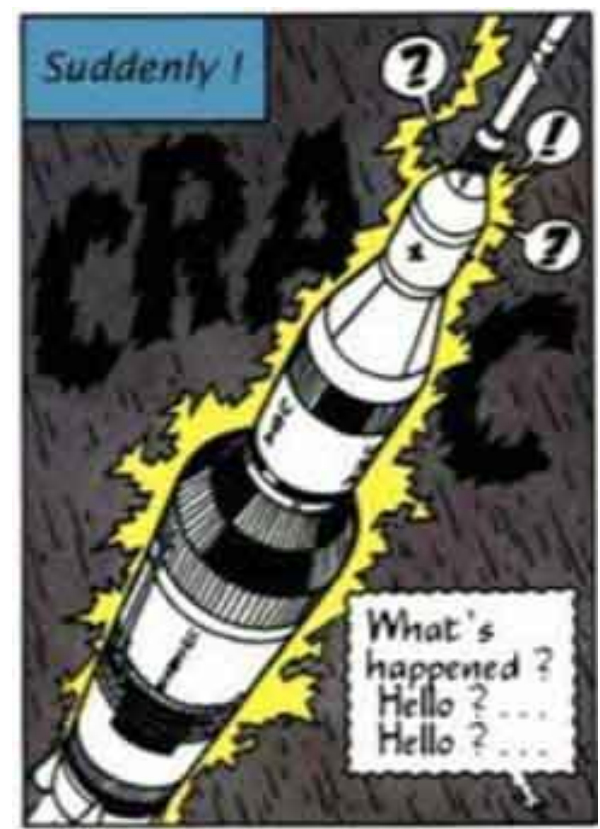

Fig. 3 - Apollo 12 (Herge, 1969)

If we apply Kress - van Leeuwen's interactive metafunction, we can again see that all of these panels offer information to the viewer; none explicitly demands a particular response. There is however an argument to be made for the panel shown in Figure 3. If we look at the visuals, yes, the diagonal line can still be interpreted as showing movement however we also see the space rocket enveloped in a yellow glow, which could be construed as something that has arrested the movement of the object - an anti-vector that cancels the sense of movement, of narration. This is further reinforced by the lack of other visual elements - vectors - that helped to create the sense of action: the smoke plumes, the motion lines present in Figure 2 which depicts the rocket departing Earth's orbit. The last thing to notice would be related to the symbolic elements that attribute good or bad qualifiers to objects. In the first panel, the lines used to create the exhaust plume coming out of the rocket's engine is straight, smooth even; the line that engulfs the rocket here is jagged, has rough almost spiky edges.

This again works to further reinforce the state of tension, to create an emotional response in the reader-viewer. If we consider the final metafunction from the Kress-van Leeuwen model, that of composition, we again observe something interesting. That which was moving, which was the center of the narrative, the rocket, is now framed in a much lower position than in the other panels. The use of colour - the bright yellow that contrasts against the black background - also gives salience to a different element that interferes with our narrative participant: the lightning strike. Looking at the text, we can observe the adverb 'suddenly' that textually communicates the same meaning as the visual: the normal course of events is interrupted by something that happens unexpectedly. A further point of argument that this panel is intended to elicit a response from the viewer-reader could be the sentences placed at the bottom of the panel "What's happened? Hello?... Hello?..." 
would fit the demand category of the interactive metafunction identified by Halliday. In other words, when considering the interactive metafunction, the visuals and the text both demand a response from the reader-viewer; they serve to create an emotional response to the apparently interrupted flight of the rocket which may or may not have devastating consequences within the overall narrative.

So far the discussion has focused on the functional aspect of images and text and it has been observed that in this particular case, the visual and the verbal elements are congruent as they each operate in similar types of metafunctional structures in conveying meaning. This is ideal from the ELT perspective, especially when this particular text is used with lower proficiency readers because text and image communicate the same message. It is important to remark however that while this is a creative text, its purpose was to be factual and to offer readers information about a real event that took place at the time of its creation. Considering its placement in a news magazine, it should not come as a surprise that its content reports an event, offers information and that visuals and text are sending the same message.

\subsection{A Structural Analysis of Comic Panels}

Moving on to a structural analysis of the visual discourse of the Apollo 12 comic, the first thing to be noticed is the use of line which creates a rather complex visual representation of the elements depicted. If we consider the characters, the objects and tools they interact with as well as the backdrops, it can be said that all these visual representations fall nearer to the realism side rather than the cartoony side of McCloud's picture triangle. What this means is that the visual message is processed a lot faster than if the visuals were more abstract and at the same time the drawing is also objective and very specific. Apollo 12 is the story of the three astronauts, Conrad, Gordon and Bean, and their lunar mission; it is not something that the reader can identify with

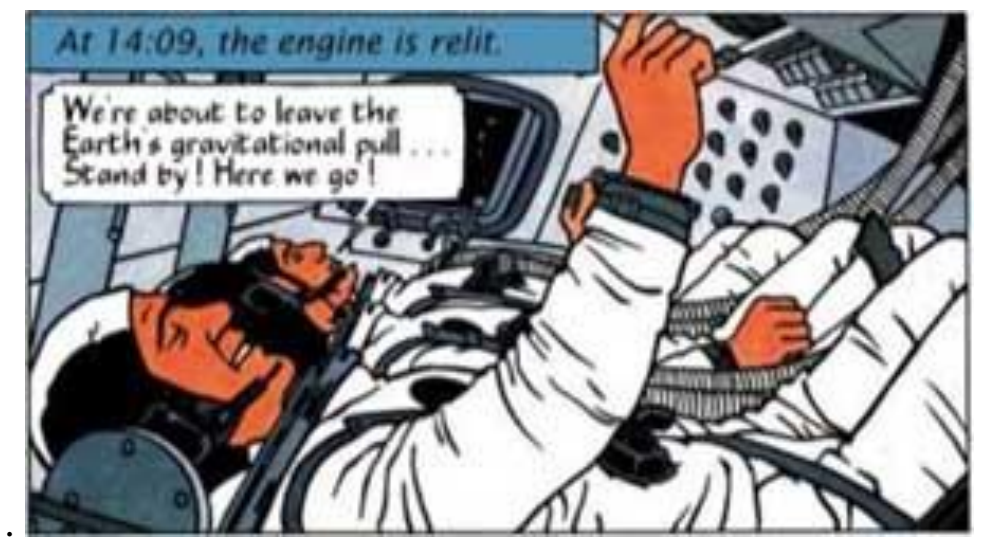

Fig. 4 - A rather realistic depiction of characters and objects (Herge, 1969)

Now contrast this with panels from Persepolis. Here the exact opposite happens. Satrapi uses a style that is closer to the iconic abstraction side in McCloud's pictorial vocabulary triangle 


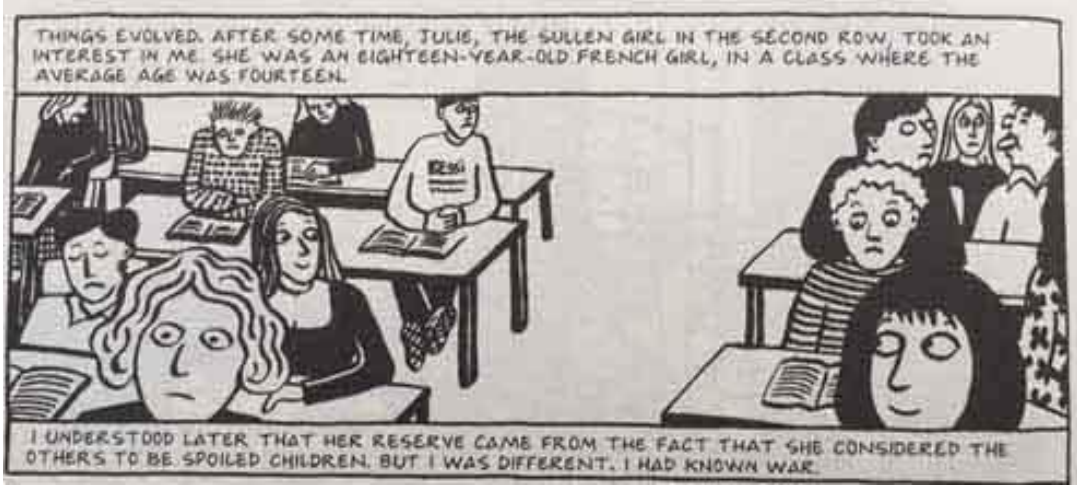

Fig. 5 - A more cartoony depiction of characters and objects (Satrapi, 2004)

The effect is reversed: the setting shown in Figure 5 can be any classroom, the faces become more universal and this in turn helps the reader viewer identify with the images. There is a second effect happening in Persepolis as a result of this visual style: because of this more abstract cartoony style, it is easier to talk about some of the difficult topics this graphic novel deals with.

\subsubsection{Open and Closed Lexical Items}

Continuing the examination of the visual morphology of comics, the focus is now shifted towards the elements that Cohn considers to be open and closed-lexical items. Elements that Cohn terms open-class lexical items (hands, eyes, face, etc.) can be combined or altered in order to create different interpretations as can be seen in the different approaches to drawing the characters in the two comics discussed in the previous section and in creating a certain style. Moving on to the closed lexical items and examining the panel shown in Figure 14, we can observe two types of speech bubbles: one created using a thin line, another created using a thinly jagged line. This difference in how the carriers are shown visually helps the reader-viewer understand who is doing the talking in this scene and where the sound - the speaker's voice - is coming from.

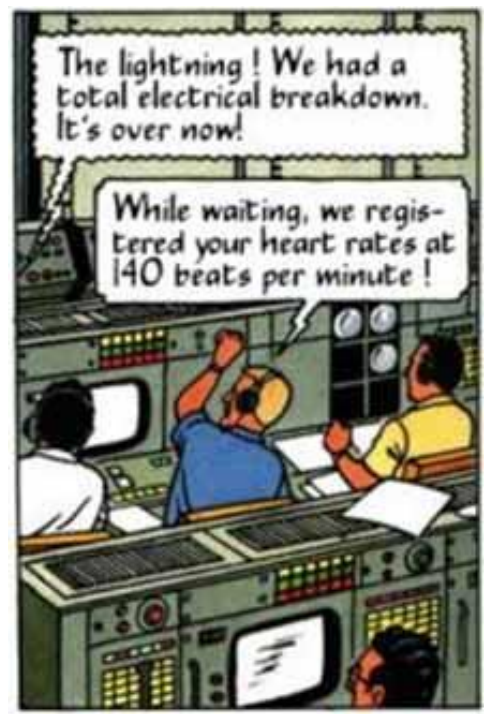

Fig. 6 - Examples of 'carriers' in Apollo 12 (Herge, 1969) 
Another category of closed-lexical items in the visual morphology of comics are the indexical lines. If we look at the two comics, Apollo 12 and Persepolis, it can be observed that, while their overall art style is different, the elements that in the chosen panels represent motion lines (Figure 2 and 7) don't change, they look and function in the exact same way; and if we look at an example of a Japanese manga (Figure 8), the same thing can be observed.

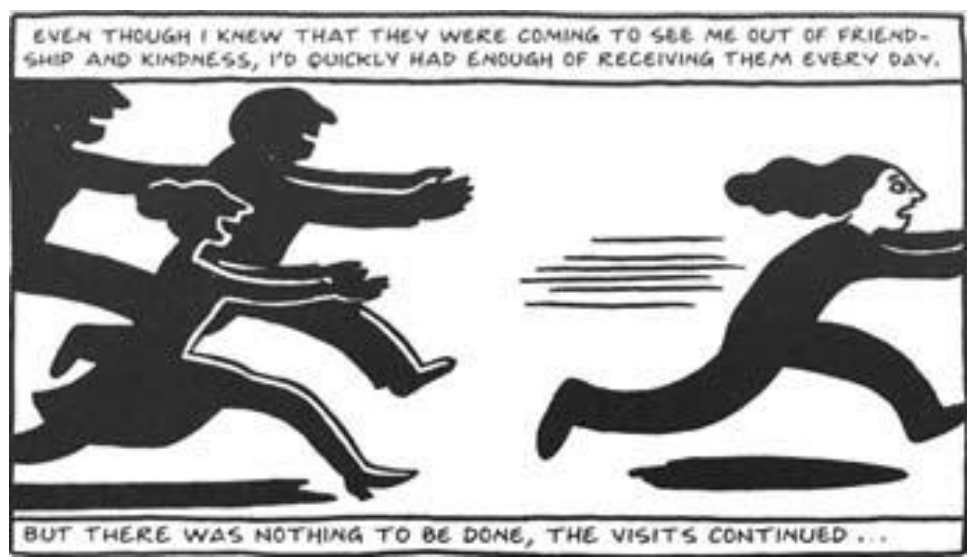

Fig. 7 - Indexical lines (motion) in Persepolis (Satrapi, 2004)

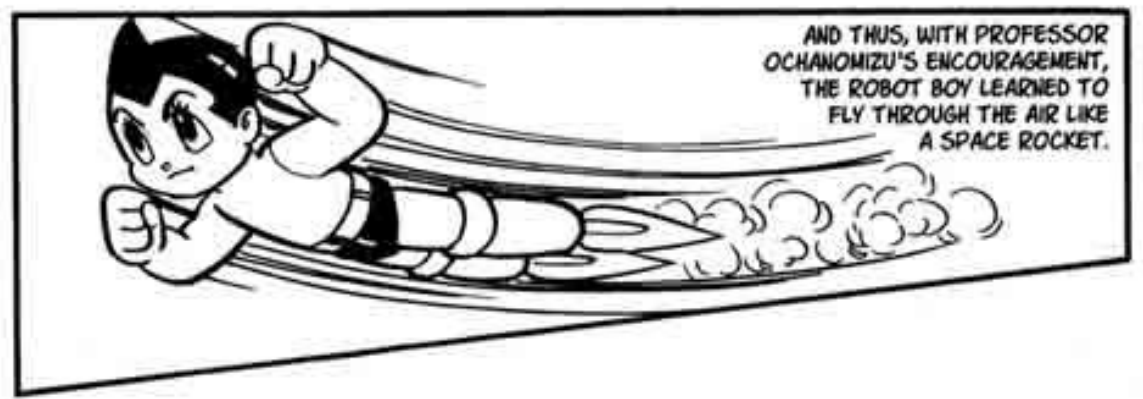

Fig. 8 - Indexical lines (motion) in Astroboy (Tezuka, 1975)

Lastly, if we look at another type of closed-lexical items we again see they fulfill the same function in the visual lexis of the comics medium regardless of the type of comic. Let us examine the Apollo 12 panel in Figure 3 and the two panels from Persepolis in Figure 9. In the Apollo 12 panel we see the two types of punctuation marks (exclamation and question marks) that visually serve to reinforce the element of surprise and confusion at what has happened in the narrative: it was unprecedented for a rocket to be struck by lightning during liftoff! In the right panel of Figure 9, Satrapi uses the same type of upfixes to suggest similar feelings of surprise and bewilderment the characters are experiencing at the sudden departure of their friend. In the left panel we can see another example of an upfix, the lightbulb above the character's head. This is a type of upfix that symbolizes thinking processes, in this case an inspiration. In this particular case, the text also reinforces the meaning of the image: "My mother had a great idea". 

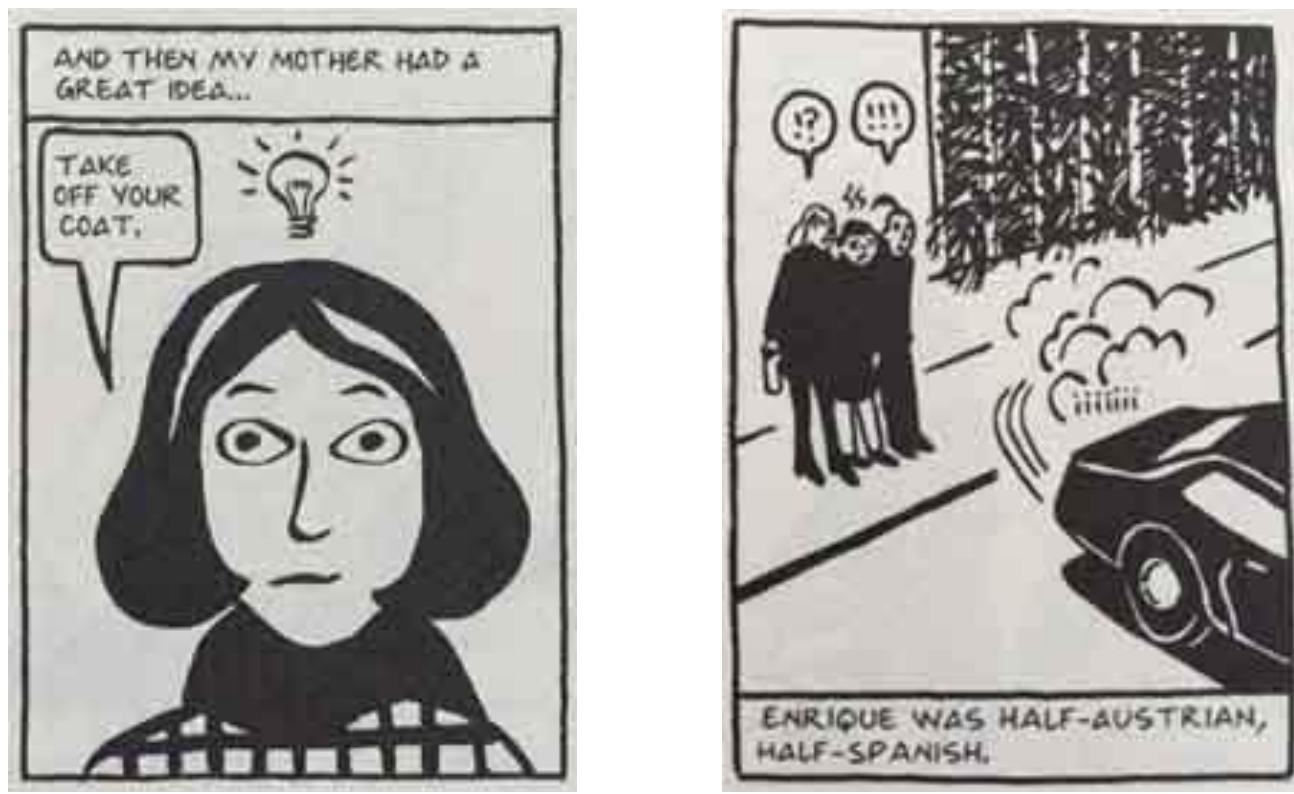

Fig. 9 - Upfixes in Persepolis (Satrapi, 2004)

Cohn calls these elements upfixes and they are found above the characters' heads to signify emotions or thinking processes. Usually these elements are conventionalized and, like the carriers, they too can be universal and have the same meaning regardless of the culture that uses them in the comic medium. The implication is that these elements again can help the comprehension of what is seen and read on the page because these elements are stored in the universal visual language system.

\subsubsection{Using the Narrative Structure}

Let us now apply the narrative structure and examine how meaning is created in the following arc from Persepolis shown in Figure 10. This series of panels tells the story of how the character got sick as a result of being homeless. There is a choice being made here that affects the way meaning is conveyed and thus the narrative pace is influenced and a sense of tension is created. If we look at the 9 panels in Figure 10 and we use the visual grammar categories created by Cohn, we can say that panel 1 works as an initial. This panel sets up the events to follow. Visually, we see the character lifting her hand to her mouth, in preparation to cough. The next panels, from 2 to 7 , also work as initials because the same information is shown: the character keeps on coughing. The use of this repetition here creates a sense of tension (the character's suffering) but also to create a sense of pace, a beat that counts down towards the inevitable conclusion. Panel 8 is the peak of the sequence where we see the result of the action that begun in panel 1. The character is now coughing blood. The last panel, 9 , is the release of this phase where we see the coda of the action, the character passed out. 
Visual Discourse of Comics in English Language Teaching
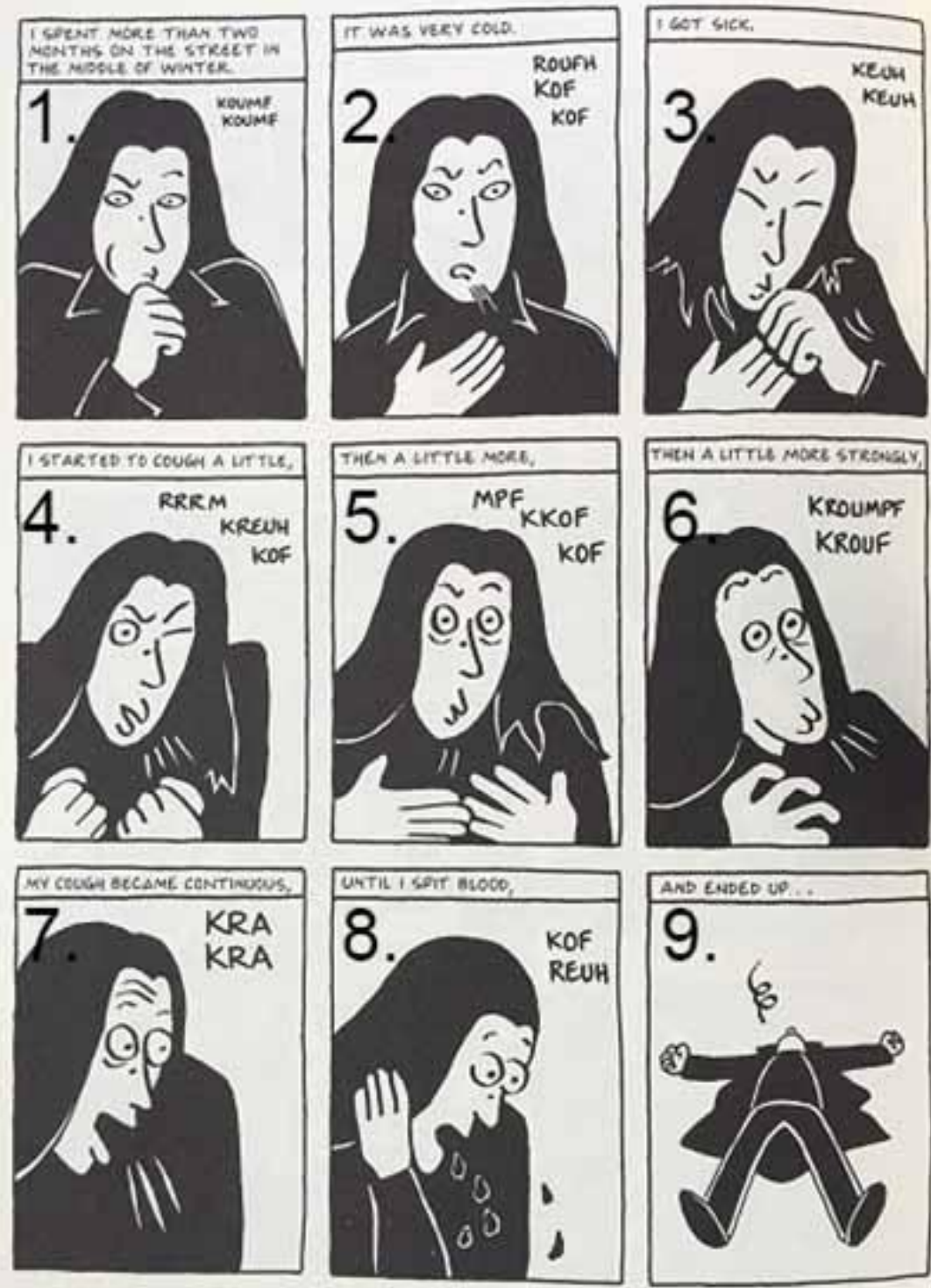

Fig.10 - Narrative structure in Persepolis (panel numbering added)

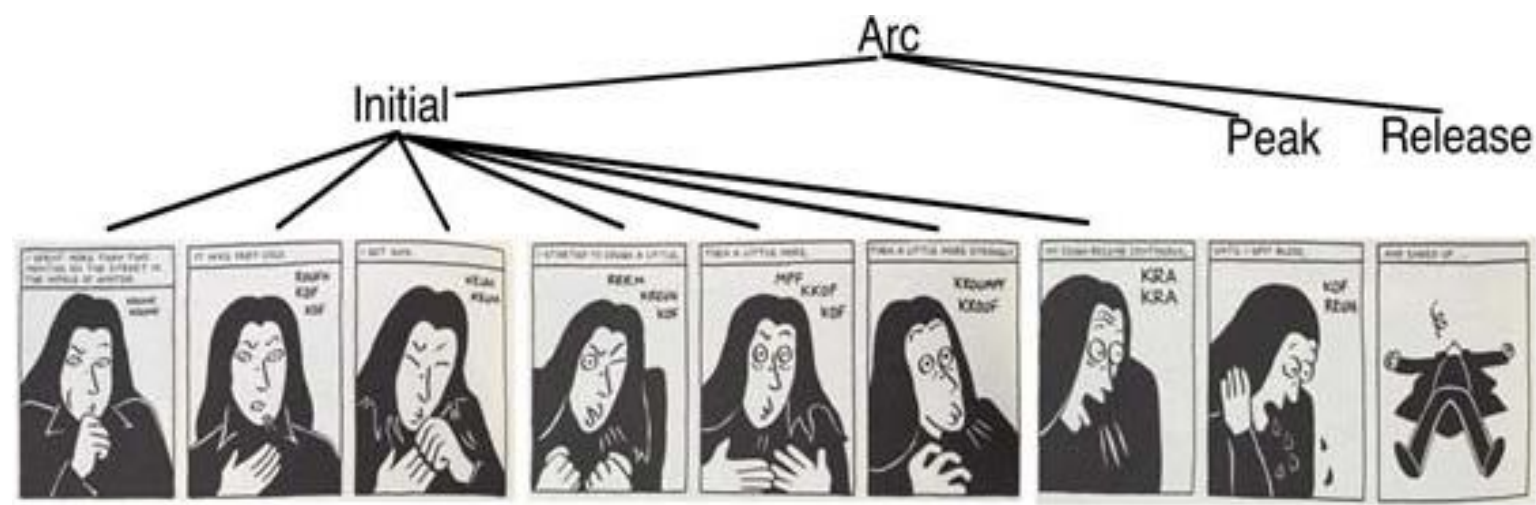

Fig. 11 - Narrative structure in Persepolis 
Breaking it down, the whole sequence then would look like the diagram in Figure 11 and it can be paraphrased according to the rules discussed previously and the results can be seen in Figure 12.

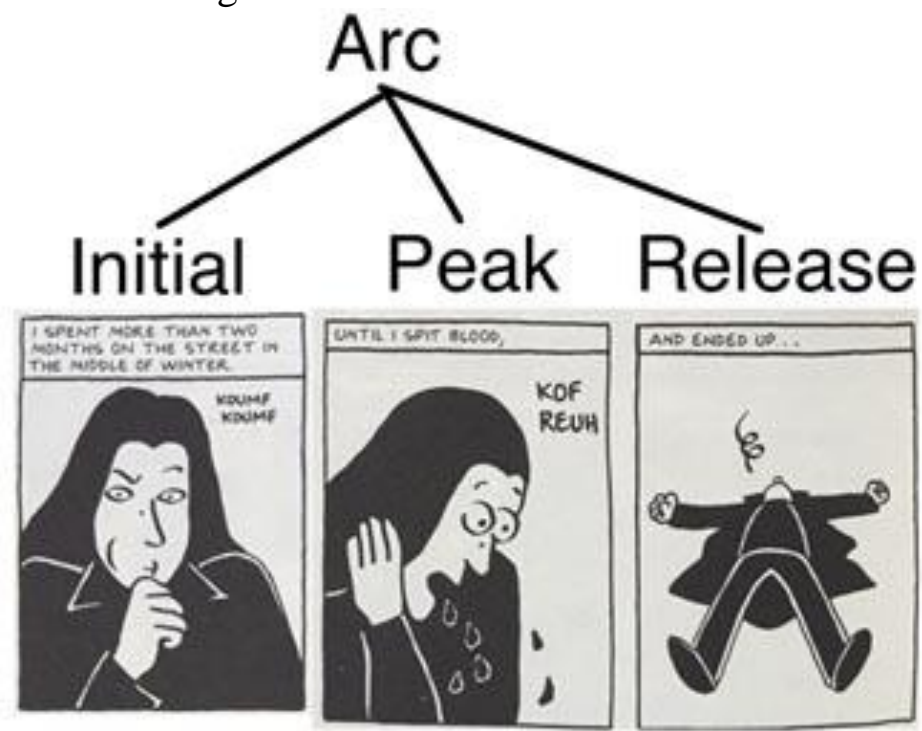

Fig. 12 - Paraphrasing of the narrative structure in Persepolis

The practical application here for ELT is that using visuals, an educator can facilitate a better understanding of the way narratives are built and the way clauses function in language.

\section{DISCUSSION}

So far it has been shown how the theory can be applied in practice using a small selection of panels. What then can be inferred about the role of the teacher, the role of the student and the role of language in a teaching approach that is based on the visual discourse of the comics?

\subsection{Language}

Looking at language, a distinction has to be made: comics are written in two systems: visual language and literary language. In the proposed framework both systems of language conform to similar ideas: the Hallidayan model for language which states that language is a system of meaning potentials based on choices dependent on context and social interactions, and Kress-van Leeuwen model of a visual grammar that is itself based on Halliday's systemic functional grammar. At the same time, in terms of structure, similarities have been identified between how a visual and a literary sentence are constructed and how there are different choices available depending on the intended meaning or message. Therefore, when using comics the educator and the learners work with two very different language systems that however operate in similar ways.

So what is language then in the proposed framework? It is a medium for communication and social interaction that is dependent and influenced by context and culture. There isn't necessarily an overt transmission of knowledge grammatical rules for example - taking place when dealing with, or learning 
language this way, but instead all factors involved in the act of communication are observed, be it verbal or visual. For example a student learning a new language following the proposed teaching approach would look at the image and at the text and simultaneously identify the possible meanings within the two systems.

Let's suppose the learner looks at panel shown in Figure 13. According to the model put forth by Kress this is an example of a narrative panel. There is an action: looking at and pointing towards the TV, which shows that the central piece of information is centered on the TV. The verbal aspect also reinforces the same idea: "There is the proof"; the text and the visuals offer similar types of information and this helps understanding.

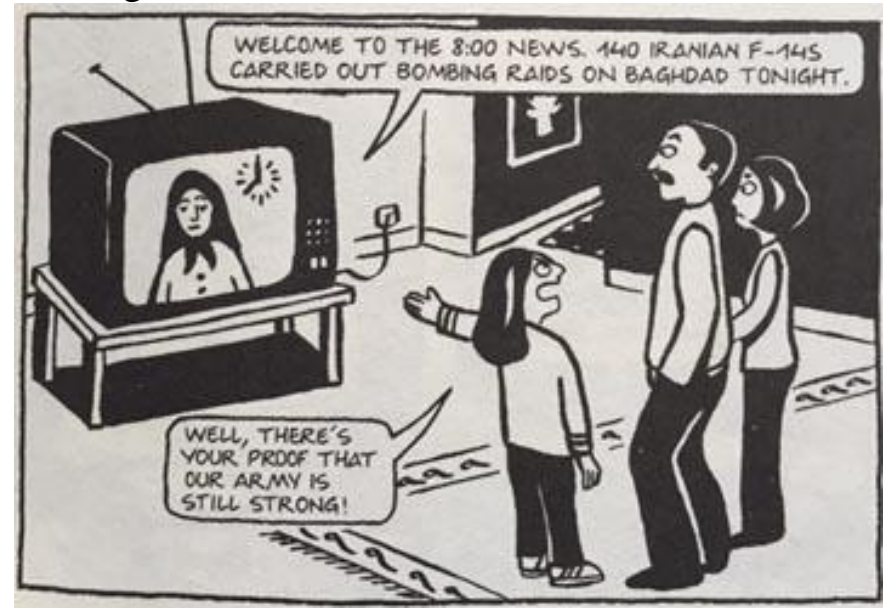

Fig. 13 - Persepolis (Satrapi, 2004)

If we look at the panel shown in Figure 14, we again see the same interaction between the two language systems. The action, the tension and sense of urgency, is seen both in the visuals - the diagonal line formed by the stairs and by the participants' hands - and in the textual: 'Let's go home now!" plus the use of the word 'terrified'.

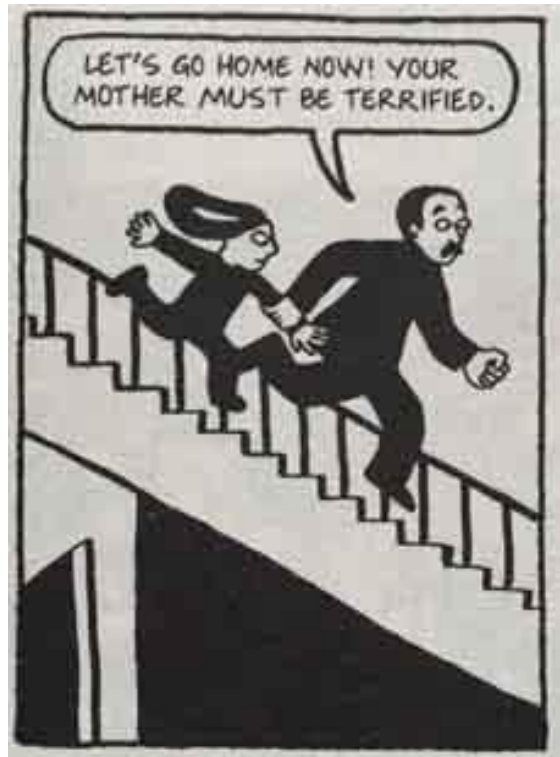

Fig. 14 - Persepolis (Satrapi, 2004) 
Let's examine what could be described as a conceptual panel. Each of the 4 panels in Figure 15 do not describe an action, instead they serve to classify the participants according to an implied taxonomy. Again, this is done both visually and verbally; the two systems of information are congruent.
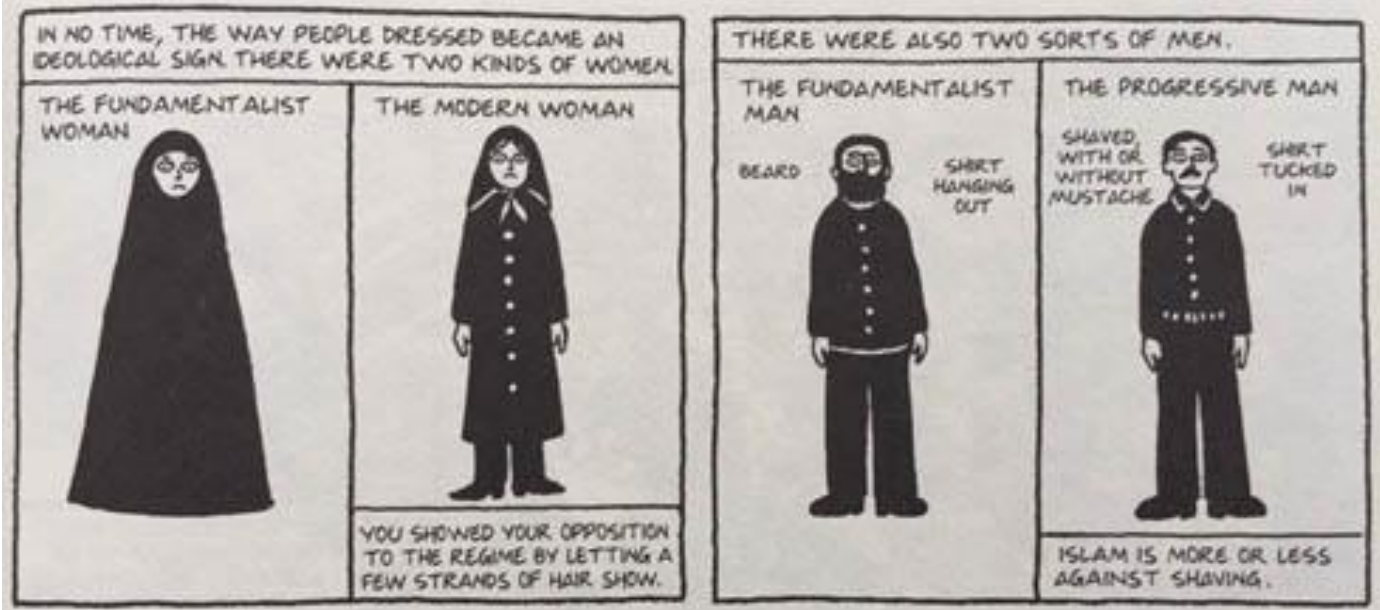

Fig. 15 - Persepolis (Satrapi, 2004)

When visuals and text are conveying the same message, visuals support reading comprehension in an L2 setting.

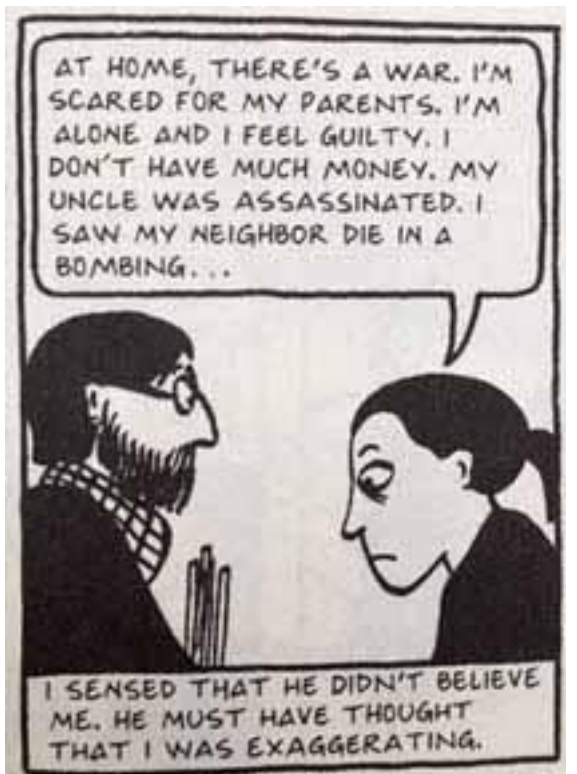

Fig. 16 - Persepolis (Satrapi, 2004)

Unfortunately for a language educator, comics, because of their very nature as authentic creative text do not only contain panels where the visual and textual information are concurrent. The overall message is a result of the interaction between text and visuals, not just a restating of it in a different system. Take the panel in Figure 16 as an example. In situations like these, from the pedagogical standpoint, comprehension is affected. 
Of course, this is just looking at and reading one panel isolated from the context of the comic and in a real classroom environment, where the whole comic would be available to the learner maybe the issue would be mitigated.

\subsection{Implications for the Teachers}

For this teaching approach to work, first and foremost the teacher needs to be consciously aware of how visual communication works, not just from the functional perspective but also from the structural one too. Apart from the functions described in the examples above, structural elements that Cohn identified as open and closed (visual) lexical elements need to be known to the teacher. The educator then must also be visually literate.

The proposed framework for using comics in the ELT classroom will enable teachers to instruct students in the interpretation and use of visuals thus answering recommendations from research carried out by Skorge (2008) or Goldstein (2016) who states that:

it is up to those who mediate the learning process, such as language teachers and materials writers, to access motivating material and to ask the right kinds of questions about it in order to engage learners and tap into [visual] literacy [...] Language learning today has to take into consideration that the messages we receive on a daily basis do not just arrive to us via the written word but in multimodal ensembles that need analyzing and unpacking. (Goldstein, 2016, p. 11)

The teacher would be a guide and a facilitator that would not point out rules or dictionary definitions but would guide the learner through the processes of meaning making that relates to the Hallidayan model for example. Also, because comics are authentic creative texts, the teacher needs to make a conscious decision when selecting the materials. Comics appeal to and are created for all ages and as such age appropriateness needs to be considered because of subject matter, complexity, sociocultural relevance and so on. A point to consider here regarding curriculum development is that because the learning materials used are comics, learners may already be familiar with this genre and may already have preferences or even their own selection already available (though not necessarily in the target language). The learners can make their own selection and this will ultimately lead to a more engaging, fun learning experience because it will not be something imposed on them.

\subsection{Implications for the Students}

How do students fit in this teaching approach? Learners are exposed to authentic, creative materials and thus they are able to experience the full spectrum that language (textual and visual) has to offer. Compared to other more traditional approaches learners observe how language is used in a real communicative situation, albeit in one type of genre - comics. Learners work independently when they read the text however once this has been completed there is room for interaction and 
discussion of either the comic or the content - or both - which leads to further language production.

\subsection{Practical Applications}

Let us briefly look at how a few practical ELT lessons would look like based on this teaching approach using the Apollo 12 comic discussed previously as well as a selection from Tezuka's Astroboy.
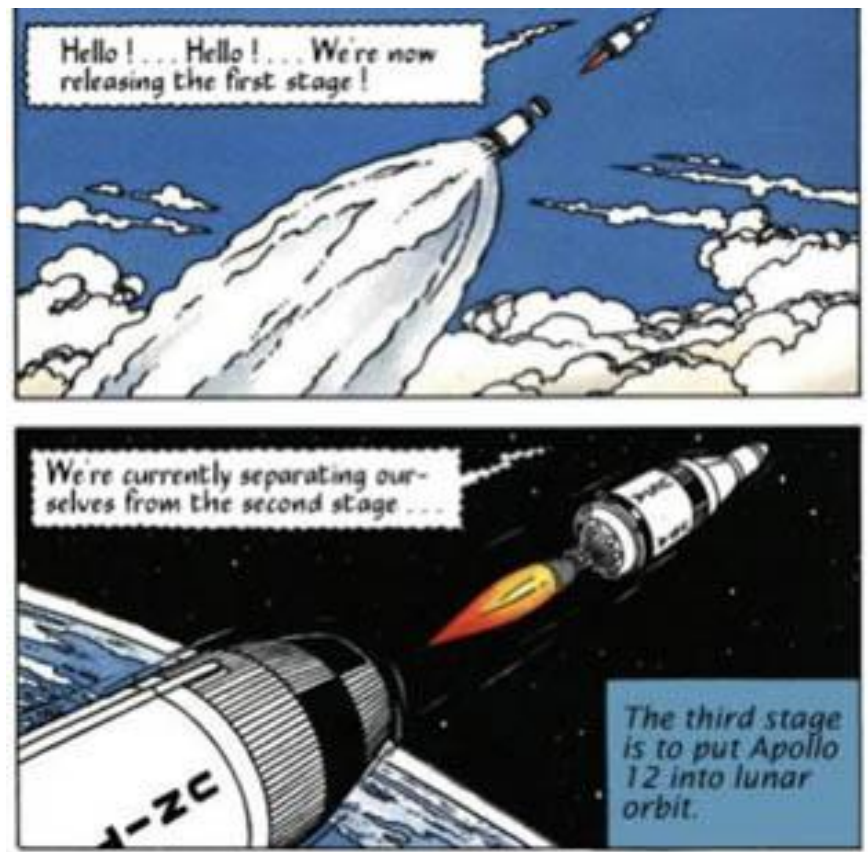

Fig. 17 - Apollo 12 (Herge, 1969)

The educator can use the panels in Figure 17 to illustrate the meaning of and the reason for using 'present continuous' in a context. It is still common for educators when teaching grammar rules to identify the rule and then to represent the tenses using a timeline that shows 'past - present - future' and the names of the tenses anywhere in-between. This can be rather confusing for several reasons: it is abstract and it lacks an immediate context. Using this comic and specifically the panels shown above, students have a context for the usage of this type of tense and are able to visualize the meaning of 'present continuous'. The literary language tells the reader-viewer that the astronauts are performing an action and are reporting it to the control center while the visual language shows the same thing: the rocket is detaching the spent fuel stages. The motion lines show precisely this - an action happening (closed class lexical items whose function is fixed and universal therefore it can aid with meaning making and understanding) and the panel is a narrative image - it tells something is happening, we have a vector for the action. So while it has been stated previously that there are no overt grammar rules transmitted using this approach to teaching English, however there is nothing that prevents an educator from drawing the learner's attention to them when and if needed especially if there is visual support for them. 
The panels in Figure 2 and Figure 17 can also be used to teach vocabulary. An ideal vocabulary lesson would not be to look up the meanings in a dictionary and memorize them but instead to look at words in context and to deduce their meaning there. Let's take for example the word 'stage'. Depending on the English proficiency of the students using this comic the literary language may or may not be enough to help learners deduce the possible meaning of the word 'stage'. The visual representation comes to the rescue here because it shows the rocket splitting up into smaller and smaller parts as it leaves Earth. Learners can guess the appropriate meaning of the word 'stage' for this context: a part of the rocket that is released when not needed anymore (when the fuel is spent).

Similarly, the visuals in Figure 2 help learners deduce the meaning of the word 'propel'. We again use the framework identified in the literature review: the panel shows a narrative image: there is an action happening, the rocket is doing something; motion lines are again present and we have the yellow and orange colors that show the exhaust flame. Put together, all these elements plus the context will lead most learners towards the appropriate meaning of the word 'propel'.

Comics can also be used in a lesson designed to help improve writing skills; this was briefly touched upon when discussing Figure 10 which showed an example of a complicated narrative structure in the graphic novel Persepolis. The panels shown in Figure 18 and 19 which belong to Astroboy can be part of a similar lesson focused on writing skills. These panels can show that the narrative is influenced by stylistic choices in both modes, visual and literary.

The teacher can start the lesson by first showing students the panel in Figure 18 (which, if we go back to Cohn's visual narrative grammar is a peak panel) and ask the learners to write a sentence based on it. The results could be similar to "The car hit the truck". Next, the teacher can introduce the panel shown in Figure 19 (which is an initial) and thus build the visual narrative.

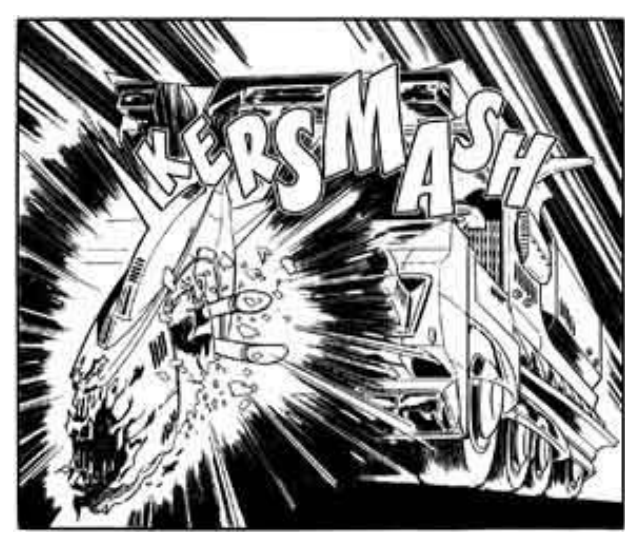

Fig. 18 - Astroboy (Tezuka, 1975)

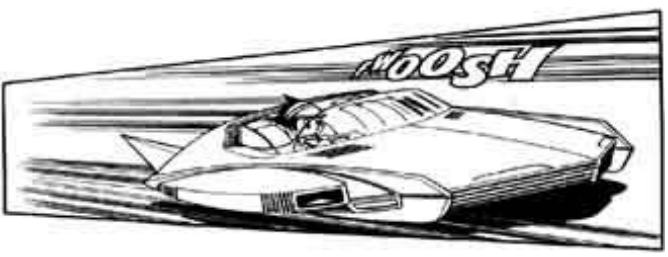

Fig. 19 - Astroboy (Tezuka, 1975)

The resulting sentence can be along the lines of "The car was driving fast then it hit a truck." The next step would be to get the learners to think about how to improve the quality of the writing by adding a sense of pace or tension to it. This is where the 2 pages of the manga shown in Figure 20 can be used: by pointing out the visual choices in building the narrative students can see why we make similar 
choices in language. The resulting piece of writing can now be more interesting: "The car was driving fast through the city, zooming through traffic, faster and faster, when all of a sudden a truck appeared from the opposite direction. It came closer and closer and then with a loud bang the two vehicles collided." (For reference, in the original manga the panel shown in Figure 18 is the last one in this arc and follows the panels shown in Figure 20.)
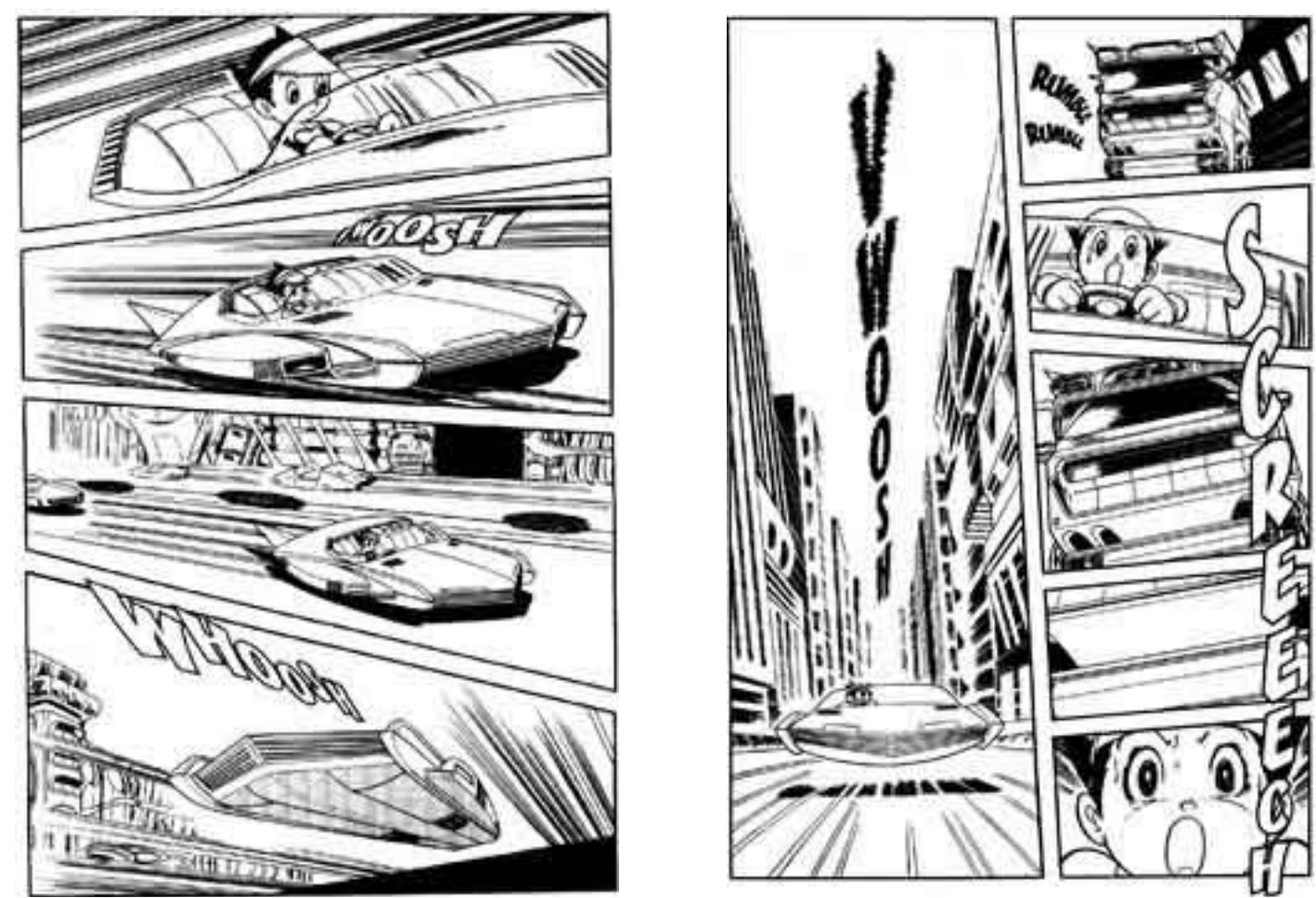

Fig. 20 - Astroboy (Tezuka, 1975)

\section{CONCLUSION}

This article proposed an ELT methodology that can be used with multimodal texts such as comics but its findings can be applied to other multimodal texts or media that follow a similar pattern (for example video games, web pages or advertisements). By using the proposed approach, a language educator will not only teach a new language but will also - ideally - teach learners to be visually literate and prepare them for a world in which information is increasingly communicated using (visual) multimodal texts.

\section{REFERENCES}

Brozo, W. \& Flynt, S.E. (2010) Visual literacy and the content classroom: a question of now, not when. The Reading Teacher 63 (6): 526-528

Cary, S. (2004). Going graphic: comics at work in the multilingual classroom. Portsmouth, NH: Heinemann

Cohn, N. (2013a). The visual language of comics: Introduction to the structure and cognition of sequential images. London: Bloomsbury. 
Cohn, N. (2013b). Visual narrative structure. Cognitive Science 34: 413-452.

Cohn, N., Jackendoff, R., Holcomb, P. J., and Kuperberg, G. R. (2014). The grammar of visual narrative: Neural evidence for constituent structure in sequential image comprehension. Neuropsychologia 64: 63-70.

Eisner, W. (1985) Comics and sequential art. Big Bear City, CA: Poorhouse.

Eisner, W. (2008) Graphic storytelling and visual narrative. New York: W.W. Norton

Goldstein, B. (2016). Visual literacy in English language teaching: Part of the Cambridge Papers in ELT series. [pdf] Cambridge: Cambridge University Press.

Halliday, M.A.K., Matthiessen, C.M.I.M. (2014) Halliday's introduction to functional grammar. Fourth Edition. London: Routledge

Kress, G., van Leeuwen, T. (2006) Reading images: the grammar of visual design. Second Edition. London: Routledge

McCloud, S. (1994) Understanding Comics: The Invisible Art. New York: Harper Perennial.

Skorge, P (2008) Visual representations as effective instructional media in foreign language teaching. Poznan Studies in Contemporary Linguistics 44 (2): 265 281 